\title{
Major adverse cardiovascular event definitions used in observational analysis of administrative databases: a systematic review
}

Elliott Bosco ${ }^{1,2^{*}+}$, Leon Hsueh ${ }^{3 \dagger}$, Kevin W. McConeghy ${ }^{1,2,4}$, Stefan Gravenstein ${ }^{1,2,3,4}$ and Elie Saade 5,6

\begin{abstract}
Background: Major adverse cardiovascular events (MACE) are increasingly used as composite outcomes in randomized controlled trials (RCTs) and observational studies. However, it is unclear how observational studies most commonly define MACE in the literature when using administrative data.

Methods: We identified peer-reviewed articles published in MEDLINE and EMBASE between January 1, 2010 to October 9, 2020. Studies utilizing administrative data to assess the MACE composite outcome using International Classification of Diseases 9th or 10th Revision diagnosis codes were included. Reviews, abstracts, and studies not providing outcome code definitions were excluded. Data extracted included data source, timeframe, MACE components, code definitions, code positions, and outcome validation.
\end{abstract}

Results: A total of 920 articles were screened, 412 were retained for full-text review, and 58 were included. Only $8.6 \%$ $(n=5 / 58)$ matched the traditional three-point MACE RCT definition of acute myocardial infarction (AMI), stroke, or cardiovascular death. None matched four-point (+unstable angina) or five-point MACE (+unstable angina and heart failure). The most common MACE components were: AMI and stroke, $15.5 \%(n=9 / 58) ; \mathrm{AMI}$, stroke, and all-cause death, 13.8\% ( $n=8 / 58)$; and AMI, stroke and cardiovascular death $8.6 \%(n=5 / 58)$. Further, $67 \%(n=39 / 58)$ did not validate outcomes or cite validation studies. Additionally, $70.7 \%(n=41 / 58)$ did not report code positions of endpoints, 20.7\% ( $n=12 / 58)$ used the primary position, and $8.6 \%(n=5 / 58)$ used any position.

Conclusions: Components of MACE endpoints and diagnostic codes used varied widely across observational studies. Variability in the MACE definitions used and information reported across observational studies prohibit the comparison, replication, and aggregation of findings. Studies should transparently report the administrative codes used and code positions, as well as utilize validated outcome definitions when possible.

Keywords: Observational study, Reproducibility, Acute myocardial infarction, Stroke, Heart failure, Acute coronary syndrome, Cardiovascular disease

*Correspondence: Elliott_Bosco@alumni.brown.edu

'Elliott Bosco and Leon Hsueh contributed equally to this work.

${ }^{1}$ Department of Health Services, Policy, and Practice, Brown University

School of Public Health, 121 South Main Street, Box G-S121-3, Providence,

RI 02912, USA

Full list of author information is available at the end of the article

\begin{abstract}
Background
Cardiovascular disease is the leading cause of death in the United States, making it a common target for interventional research $[1,2]$. Due to this, the composite endpoint of "major adverse cardiovascular events" (MACE) is an increasingly common primary outcome of interest. In 2008, the United States Food and Drug Administration (FDA), followed by the European Medicines Agency
\end{abstract}

(c) The Author(s) 2021. Open Access This article is licensed under a Creative Commons Attribution 4.0 International License, which permits use, sharing, adaptation, distribution and reproduction in any medium or format, as long as you give appropriate credit to the original author(s) and the source, provide a link to the Creative Commons licence, and indicate if changes were made. The images or other third party material in this article are included in the article's Creative Commons licence, unless indicated otherwise in a credit line to the material. If material is not included in the article's Creative Commons licence and your intended use is not permitted by statutory regulation or exceeds the permitted use, you will need to obtain permission directly from the copyright holder. To view a copy of this licence, visit http://creativecommons.org/licenses/by/4.0/. The Creative Commons Public Domain Dedication waiver (http://creativeco mmons.org/publicdomain/zero/1.0/) applies to the data made available in this article, unless otherwise stated in a credit line to the data. 
(EMA) in 2012, provided guidance on utilizing a threepoint MACE outcome, which includes acute myocardial infarction (AMI), stroke, and cardiovascular mortality in all trials evaluating the cardiovascular safety of diabetic agents [3]. Some trials have utilized a four-point MACE as well, by including hospitalization for unstable angina or revascularization procedures $[3,4]$. Five-point MACE further expands on this with the inclusion of heart failure (HF). While MACE is now a better-defined and more ubiquitous outcome among RCTs, its use in observational studies to assess the safety and real-world effectiveness of therapies remains less clear.

Observational studies using large administrative databases can evaluate population-level use and outcomes of therapies in an efficient and cost-effective way [5]. However, several common issues with observational studies limit their reproducibility and comparability to RCTs, thus limiting the utility of MACE as a composite outcome. First, in comparison to RCTs, observational studies often do not consistently report their protocols, including outcome definitions [6]. To allow for useful comparisons between observational studies and RCTs, improved standardization and transparency is needed [7, 8]. Even when MACE components are well-defined, such as AMI or stroke, another challenge encountered in observational studies is how to define outcomes using diagnosis codes available from administrative data. For example, the International Classification of Diseases (ICD) is a commonly used coding system for medical reimbursement and is one of the most frequent sources of information available in administrative databases [9]. Unfortunately, ICD codes can be prone to errors and diagnosis misclassifications, as they are primarily collected for reimbursement purposes and rely on clinical documentation that can vary across settings and providers [5]. Though several studies have attempted to validate the diagnosis codes for commonly used MACE components (e.g., AMI, stroke, and HF), with positive predictive values of upwards of $80-90 \%$, it is unclear how these codes have been taken up in MACE composite outcome definitions [10-12]. For these reasons, the current use of MACE in observational studies warrants further investigation.

Thus, the purpose of this review was to systematically determine the most common definitions of MACE employed in observational studies using administrative data. With that, our objectives were: i) assess each study's definition of MACE components (e.g., AMI, stroke), ii) assess the diagnostic criteria used for outcome ascertainment such as codes used and position of codes, and iii) assess whether outcomes had been validated. We hypothesized that, across observational studies, there is great variability in the definitions of MACE used and minimal alignment with the classic three, four, or five-point
MACE outcomes. Our hope is that this work will promote a standard approach to the definition of MACE in future studies, allowing for the improved transparency and reproducibility of observational studies.

\section{Methods \\ Search strategy and selection criteria}

The protocol for this systematic review is based on the 2015 Preferred Reporting Items for Systematic Reviews and Meta-Analysis Protocols (PRISMA-P) statement [13]. The protocol was not registered in the International Prospective Register of Systematic Reviews (PROSPERO), but the original protocol and modifications are included in Supplementary Text 1 (see Additional file 1). The reporting of this review is based on the 2020 PRISMA statement and the checklist is provided in Supplementary Table 1 (see Additional file 2) [14]. We searched MEDLINE and EMBASE for literature published from January 1, 2010 to October 9, 2020 that defined composite MACE as a primary or secondary outcome in studies utilizing administrative databases. A 10-year lookback was used in order to restrict to the most recently published studies. The search strategy was developed by the authors using the search terms presented in Supplementary Text 2 (see Additional file 3) and was performed on October 9, 2020. A standardized protocol for study abstract screening and full-text review was developed and piloted using the same 20 studies among the reviewers (EB, ES, LH), and is further described in Supplementary Text 1 (see Additional file 1). The abstracts from each record were initially screened for relevance by three independent reviewers (EB, ES, LH) using Abstrackr (http://abstrackr.cebm. brown.edu/), a free semi-automated abstract screening tool [15]. Afterwards, we performed a full-length text review of the abstracts that were retained after screening (EB, ES, LH).

We included studies that identified MACE composite endpoints as the primary or secondary study outcome, used administrative data sources in the study, and defined MACE using International Classification of Diseases, Clinical Modification, Ninth Revision (ICD9-CM) or Tenth Revision (ICD-10-CM) diagnosis codes. The included studies did not need to overtly name "major adverse cardiovascular events" as the primary or secondary outcome to be included into this study, as many definitions and terms are used across studies. For example, MACE may be referred to as "adverse" or "acute cardiovascular events", "major adverse cardiovascular and cerebrovascular events" or simply "cardiovascular events", among other terms. Thus, we included studies if the composite outcome that was studied utilized a combination of multiple MACE endpoints. We excluded reviews, meta-analyses, conference abstracts, 
editorials, papers whose primary or secondary outcome was not a composite of multiple MACE endpoints, and those that did not use or report ICD-9-CM or ICD-10-CM codes to define MACE endpoints. Any uncertainty with regards to inclusion or exclusion of an individual paper was resolved by consensus of the three reviewers.

\section{Data extraction and synthesis}

After each of the independent reviewers determined that a full-text met inclusion criteria for the study, definitions for composite MACE outcomes were extracted into a shared document using a standardized protocol by two reviewers (LH, EB). ICD-9-CM and ICD-10-CM codes were also extracted for each MACE endpoint, and were acquired either through full-length text review or through review of published supplements. The position of the diagnosis code required in the study outcome criteria was recorded as primary position, any position, or not reported. Further, information on whether the study outcomes had been previously validated was assessed in each individual study or review of supplements, and citations were extracted. Each study's administrative data source and study years were also recorded.

Once data were extracted from included studies, we categorized the specific definitions of MACE based on the individual components referred to by the authors as opposed to the specific diagnosis codes used. We made this decision due to the lack of consistency in diagnosis codes used across studies and chose to categorize based on the MACE components that the authors reported. Component definitions of MACE included: AMI, acute coronary syndrome or ischemic heart disease (ACS/ IHD), stroke (either ischemic or hemorrhagic stroke), revascularization procedures, cardiovascular $(\mathrm{CV})$ death, and all-cause death. Of note, due to the variability of outcomes used across studies, the ACS/IHD component reflects the following definitions used by study authors: ACS, IHD, coronary artery disease (CAD), and unstable angina (UA). Overall, we performed a qualitative assessment of the evidence only, and did not perform a metaanalysis or strength of evidence assessment due to the nature of the research questions.

\section{Results}

\section{Included studies}

Our search of MEDLINE and EMBASE yielded 920 unique articles, 412 of which were retained for full-text review after abstract screening (Fig. 1). After excluding 354 studies during full-text review, 58 studies were included in the final analysis.

\section{Overall studies}

The included observational studies utilized a range of outcome components to define MACE (Table 1). The majority of the included studies utilized ICD-9-CM to define outcomes. There was poor concordance of MACE definitions used in the included studies when compared to the three-point, four-point, and five-point definitions of MACE commonly used in randomized controlled trials (RCTs). For instance, 8.6\% (5/58) of observational studies used a MACE definition that matched the threepoint definition of MACE (AMI, stroke, CV death) while no studies matched the four-point or five-point definitions (Table 2). Across all included studies, the most common MACE component definitions were: AMI, stroke, 15.5\% (9/58); AMI, stroke, all-cause death, 13.8\% (8/58); and AMI, stroke, CV death 8.6\% (5/58) (Table 3). Overall, $67 \%$ of studies (39/58) did not perform a validation or provided no citations validating the outcome definitions used. Additionally, 70.7\% (41/58) of studies did not report the position of diagnosis codes used in endpoints, while $20.7 \%(12 / 58)$ used the primary diagnosis position and $8.6 \%(5 / 58)$ used any position.

\section{MACE component definitions Acute myocardial infarction}

There were 45 studies that included AMI as a component of MACE (Table 2). Of these, 64.4\% (29/45) defined outcomes using ICD-9-CM and 31.1\% (14/45) used ICD10-CM. Among these studies, 20\% (9/45) defined AMI in the primary diagnosis position, $6.7 \%(3 / 45)$ in any position, and $73.3 \%$ (33/45) did not report the position used. The most common diagnosis codes were: $410 . \mathrm{xx}$, making up 72\% (21/29) of the ICD-9-CM studies; and I21.xx, I22. $\mathrm{xx}$, making up $35.7 \%(5 / 14)$ of the ICD-10-CM studies (Table 4).

\section{Acute coronary syndrome / ischemic heart disease}

There were 18 studies that defined ACS/IHD as a component of MACE. ICD-9-CM diagnosis codes only were used in $66.7 \%(12 / 18)$ of studies and $27.7 \%(5 / 18)$ used ICD-10-CM only. Studies used the primary diagnosis position $22.2 \%(4 / 18)$, any position $11.1 \%(2 / 18)$, and did not report the position $66.7 \%(12 / 18)$ of the time. The most common diagnosis codes were: 410-414.xx, 25\% (3/12) of the ICD-9-CM studies; and I21-I24.xx, 40\% $(2 / 5)$ of the ICD-10-CM studies.

\section{Stroke}

There were 50 studies that included stroke, either ischemic or hemorrhagic, as a component of MACE. Of these, $60 \%(30 / 50)$ utilized ICD-9-CM only and 34\% $(17 / 50)$ used ICD-10-CM only. The primary diagnosis 


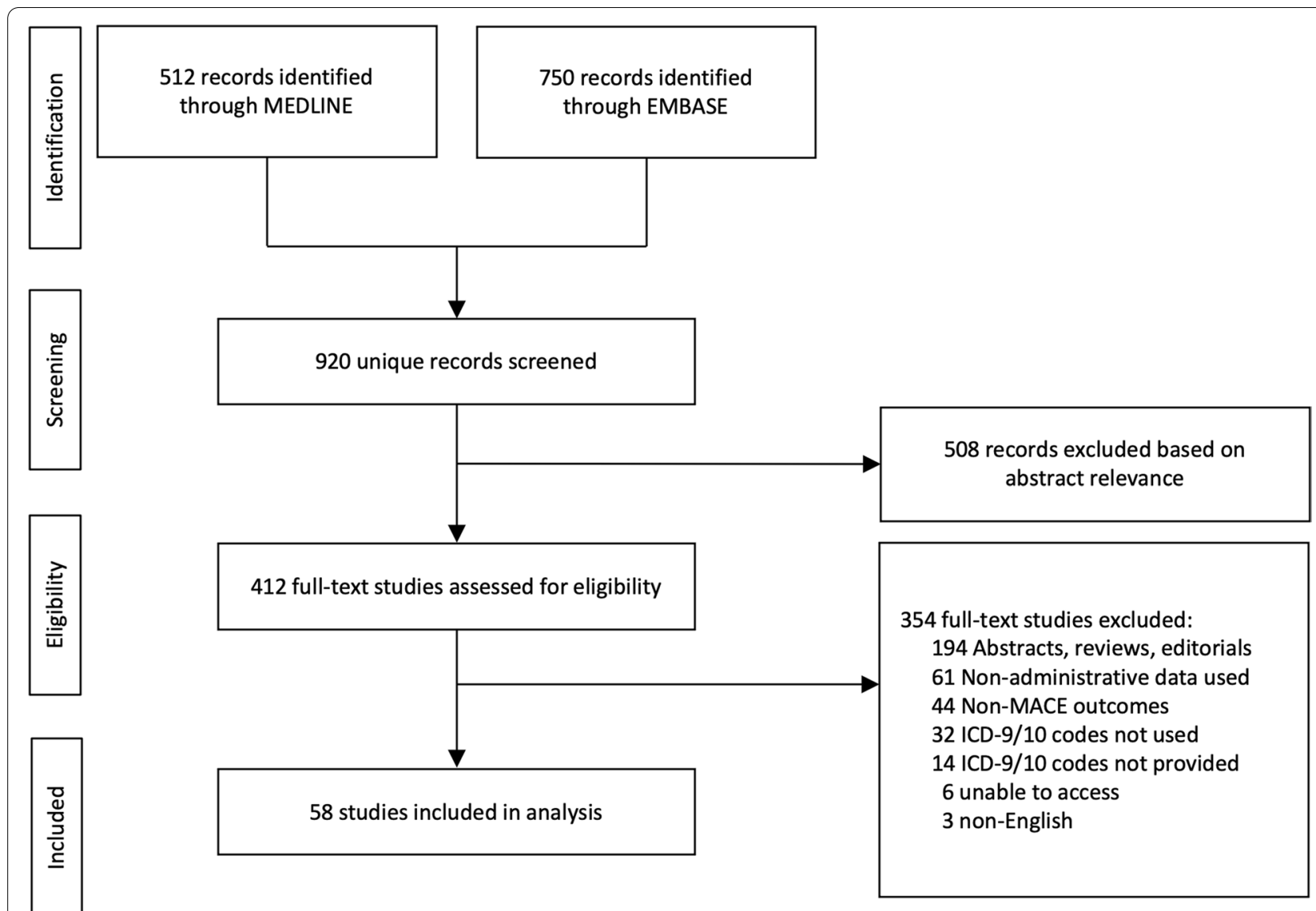

Fig. 1 PRISMA flow diagram for included studies. Abbreviations: MACE, major adverse cardiovascular events; ICD, International Classification of Diseases

position was used $24 \%(12 / 50)$ of the time, any position used $8 \%(4 / 50)$, and no position reported $68 \%(34 / 50)$ of the time. Diagnosis codes used to define stroke were highly variable with no clear most common definition. Codes included: 433.xx, 434.xx, 436.xx, 13.3\% (4/30) of ICD-9-CM studies; 430-437.xx, 13.3\% (4/30) of ICD-9-CM studies; I63.xx, I65.xx, I66.xx, 17.6\% of ICD-10-CM studies (3/17); and I63.xx, 17.6\% (3/17) of ICD-10-CM studies.

\section{Heart failure}

A total of 15 studies included $\mathrm{HF}$ as a component of MACE. Of these studies, 80\% (12/15) used ICD-9-CM only, and $13.3 \%(2 / 15)$ used ICD-10-CM only. The primary diagnosis position was used $20 \%(3 / 15)$ of the time but was otherwise not reported in $80 \%(12 / 15)$ of studies. The most common diagnosis codes used were: 428.xx, $50 \%$ (6/12) of ICD-9-CM studies; I11.0, I50.xx, I97.1, 50\% $(1 / 2)$ of ICD-10-CM studies; and I50.xx, K76.1, I97.1, I11.0, 50\% (1/2) of ICD-10-CM studies.

\section{All-cause death and cardiovascular death}

There were nine studies that included cardiovascular death as a component of MACE. Of these, 11.1\% (1/9) of studies utilized ICD-9-CM codes only for MACE components and $66.7 \%$ (6/9) used ICD-10-CM. The diagnosis position for cardiovascular death was primary $11.1 \%(1 / 9)$, any position $33.3 \%$ (3/9), and not reported $55.6 \%(5 / 9)$ of the time. The most common diagnosis codes used were: No codes listed, $100 \%(1 / 1)$ for studies using ICD-9-CM only; and I00.xx-I99.xx, $33.3 \%(2 / 6)$ for those using ICD-10-CM only. There were 15 studies including all-cause death as a component of MACE. Due to the nature of all-cause death, diagnosis codes positions are not necessary and were not reported.

\section{Discussion}

In our systematic review, we found substantial heterogeneity for MACE composite endpoints used in the literature. The two most common composite MACE 


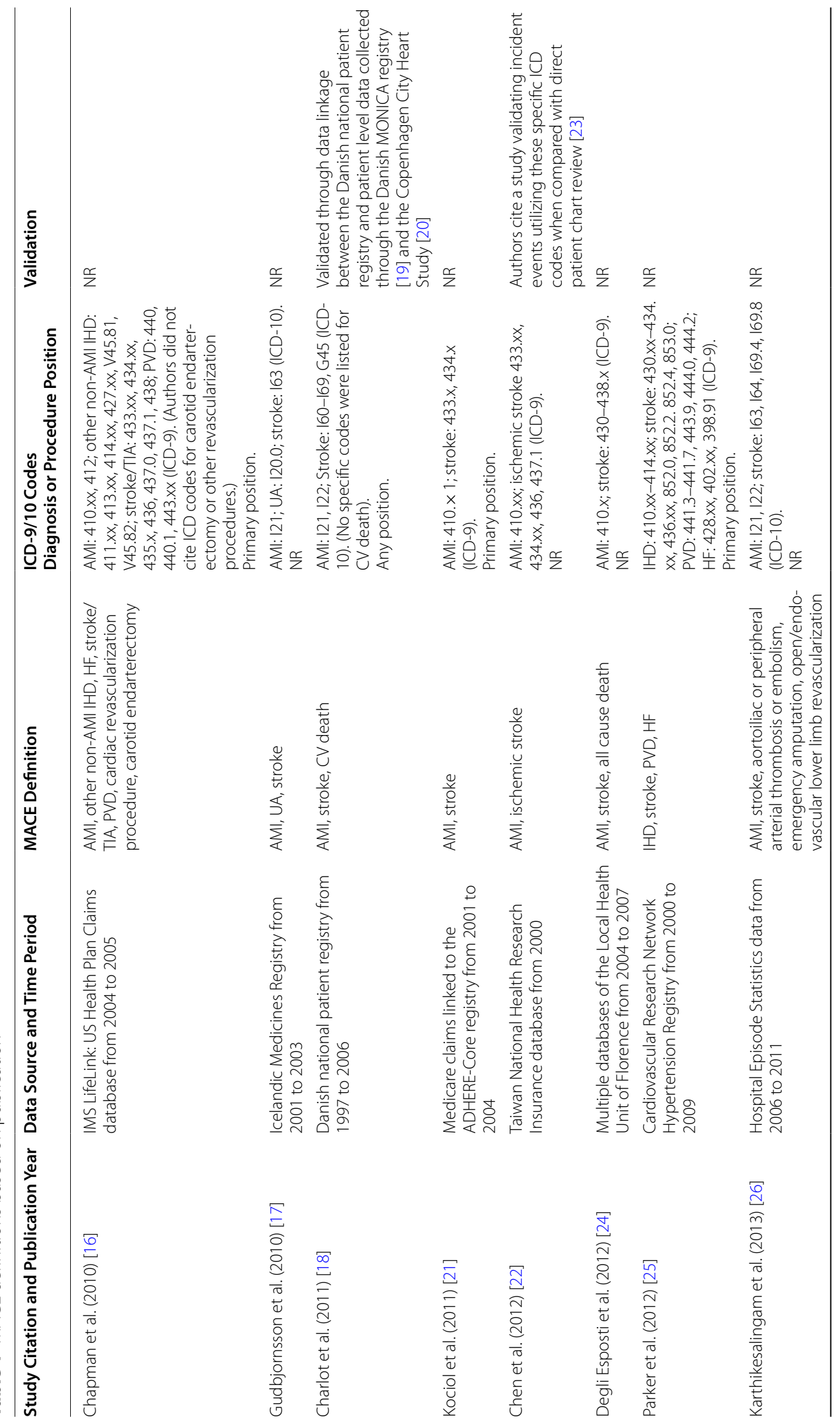




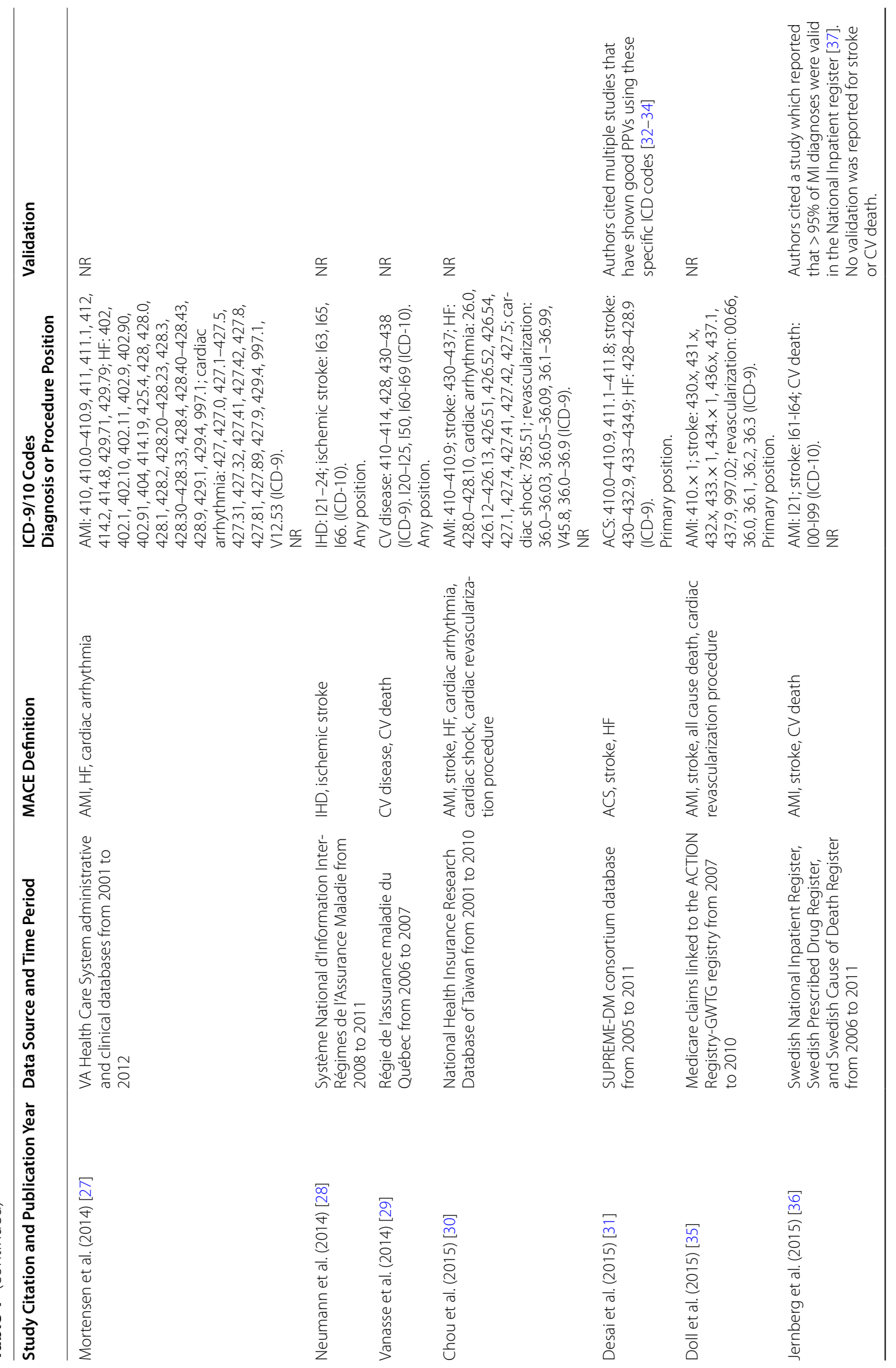




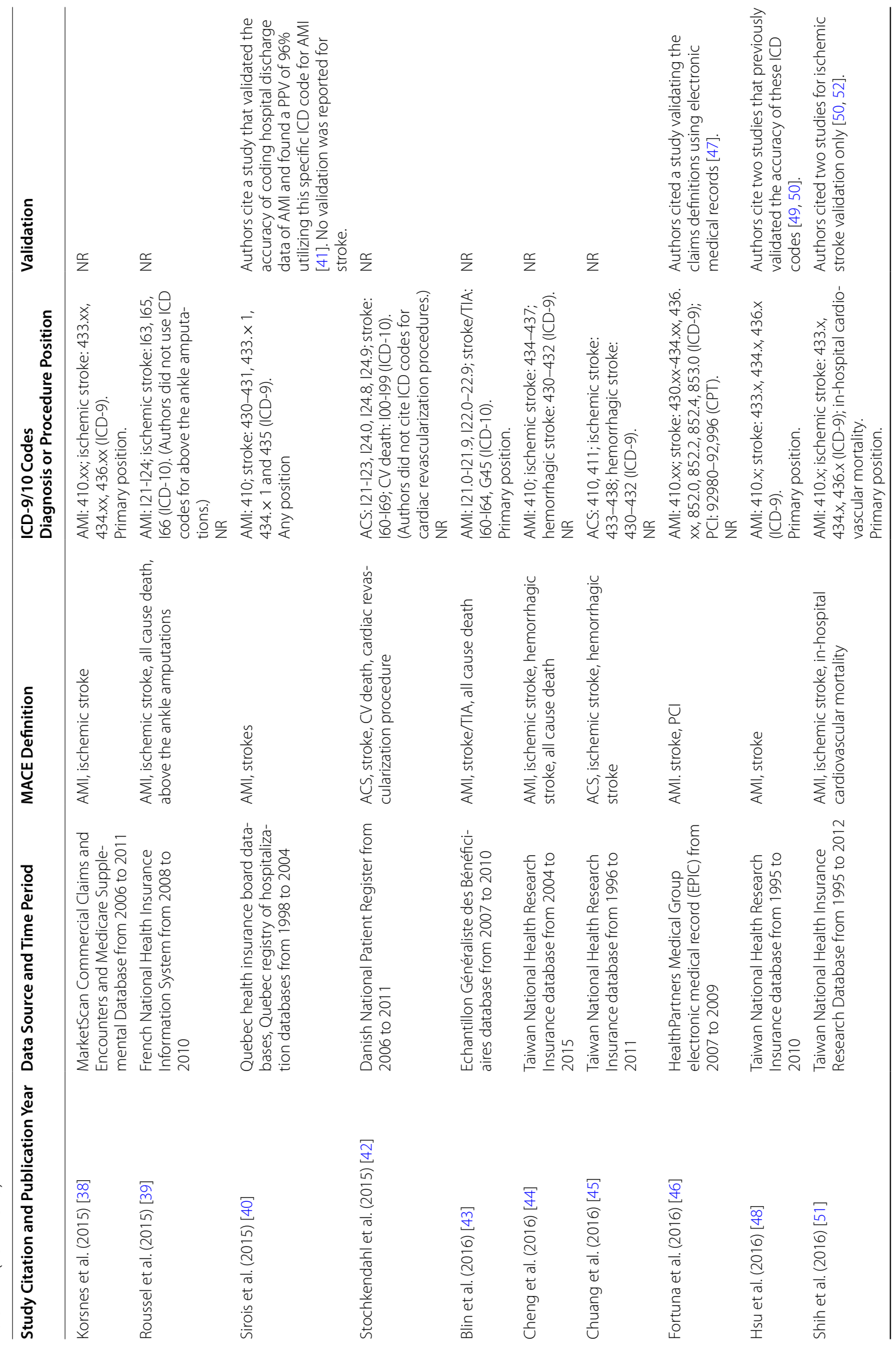




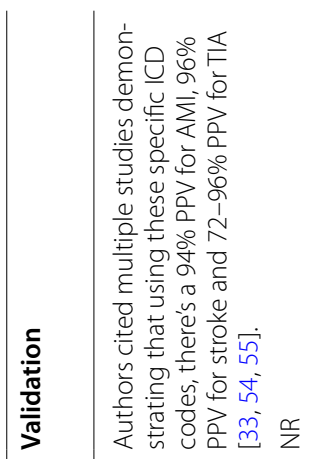

$\stackrel{\frac{c}{z}}{\frac{c}{z}} \stackrel{\frac{c}{z}}{2}$

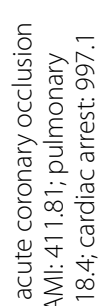

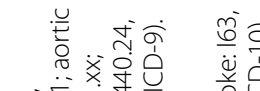

这家

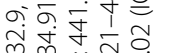

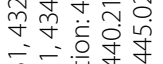

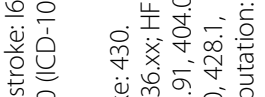

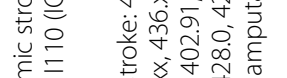

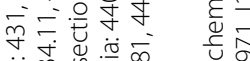

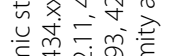

हु

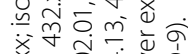

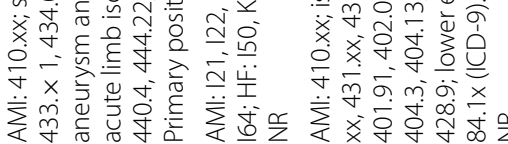

$\stackrel{\propto}{z}$

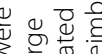

要

0
0

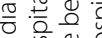

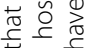

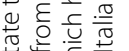

证定

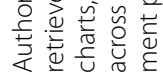

$\stackrel{\infty}{z}$

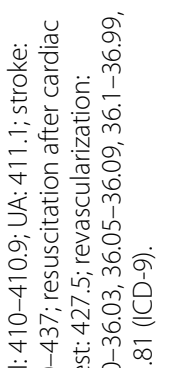

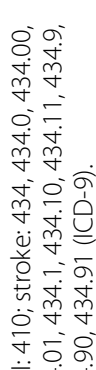

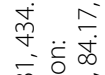

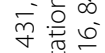

宇产市

링

产高安

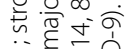

$\bar{\tau}$

xym

$\frac{7}{2}=0$

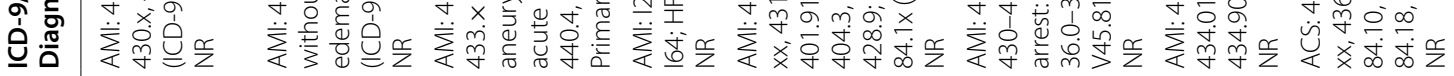

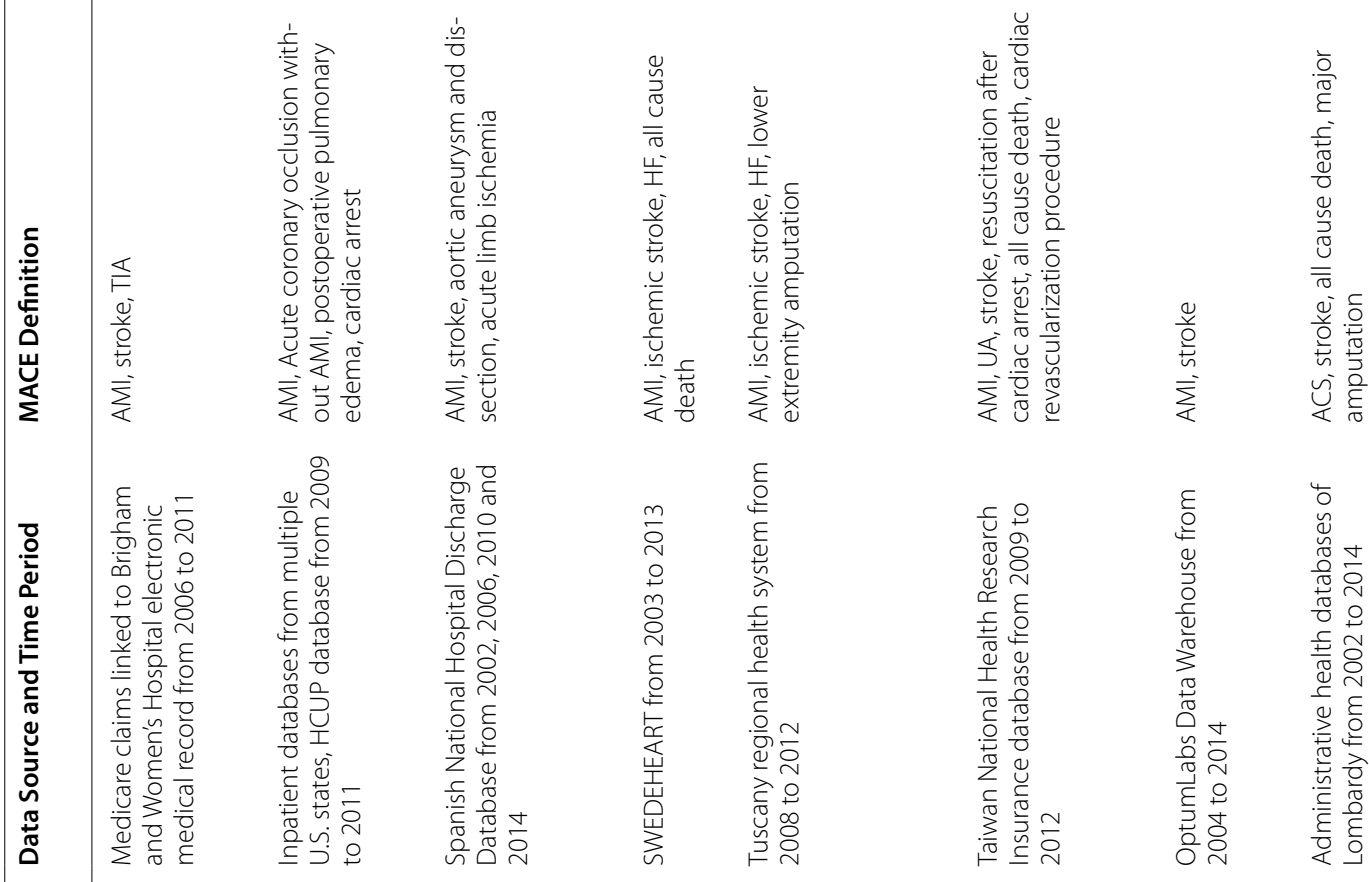

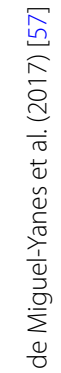

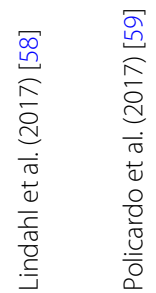

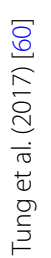

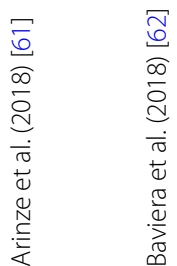




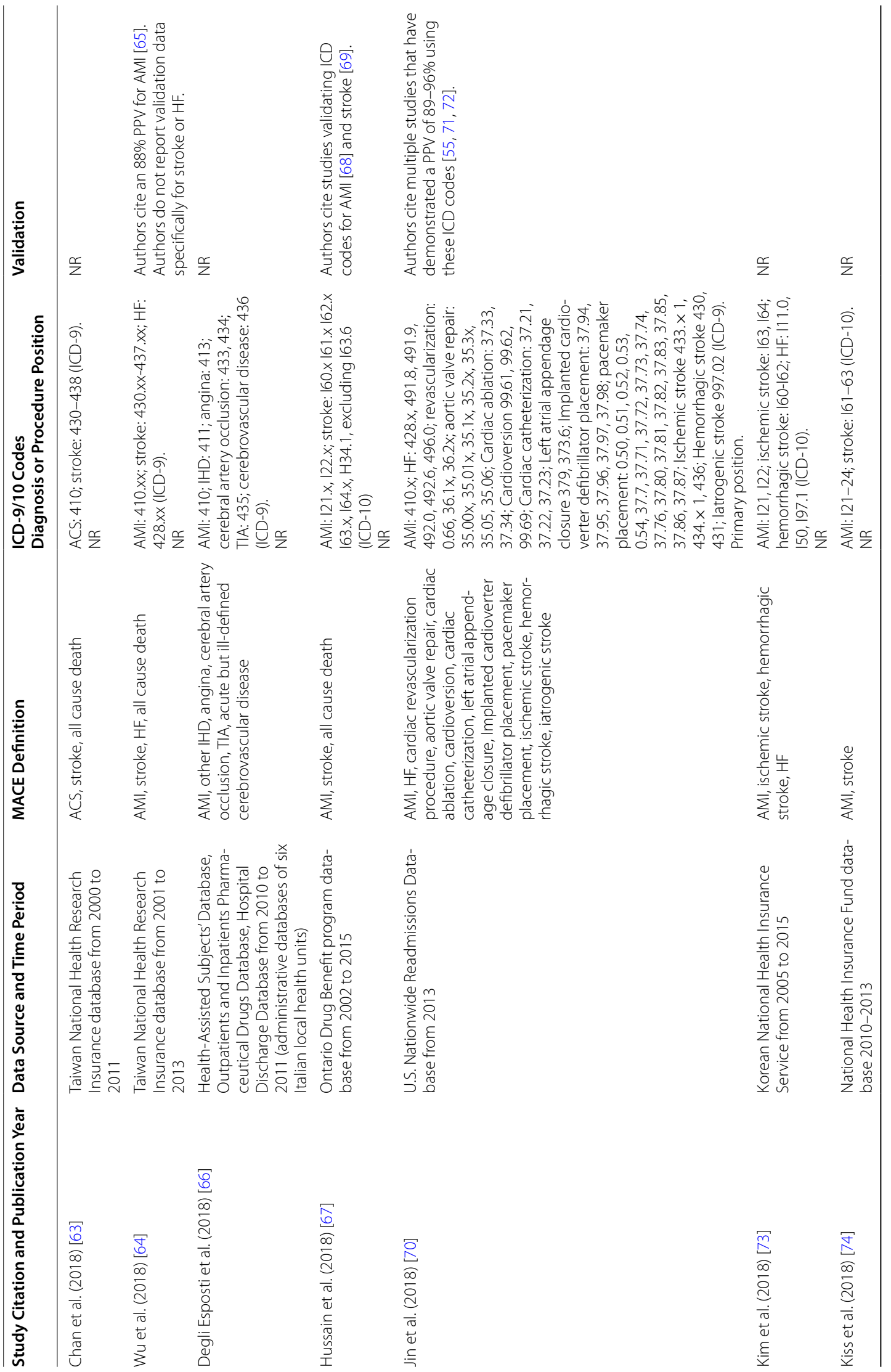




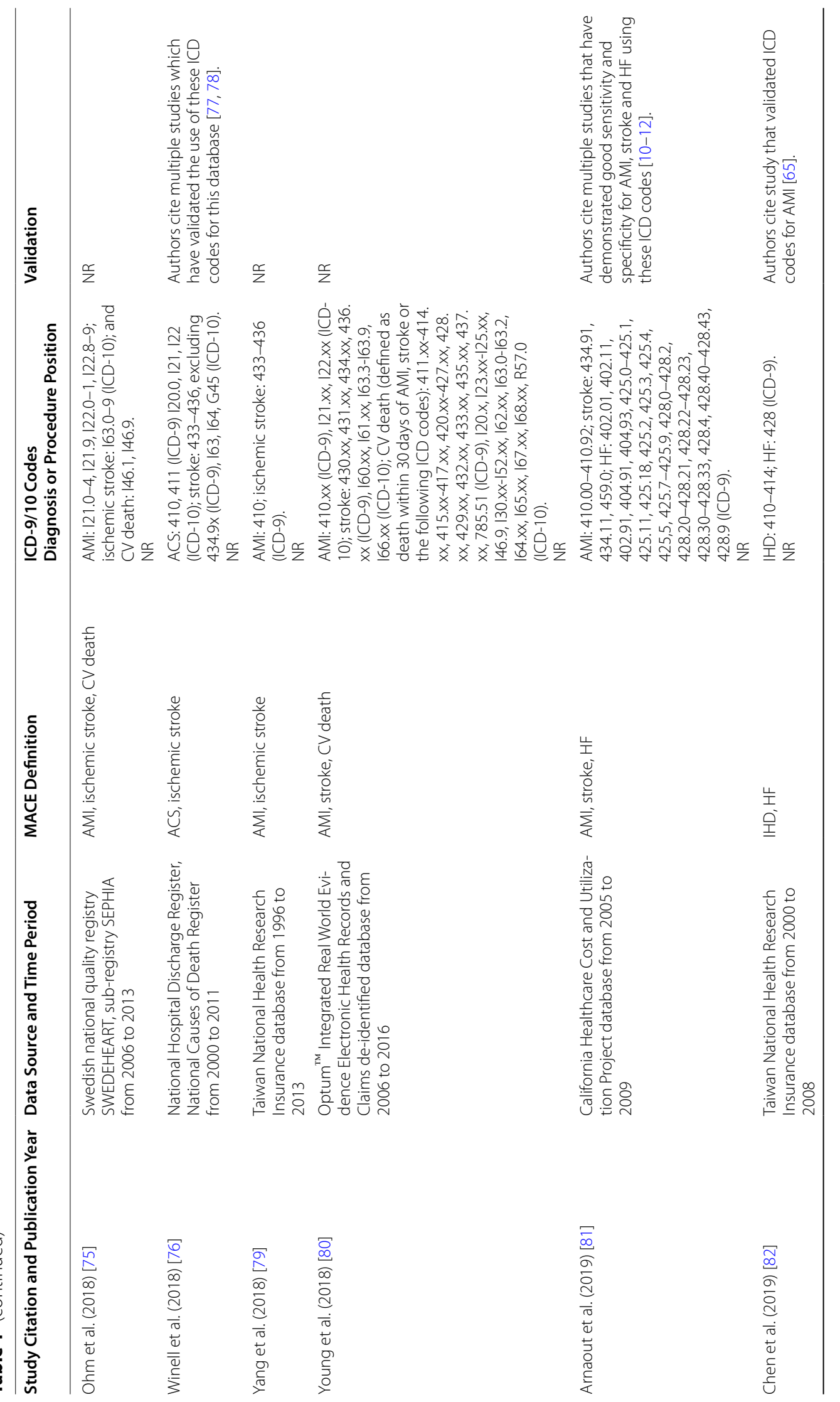




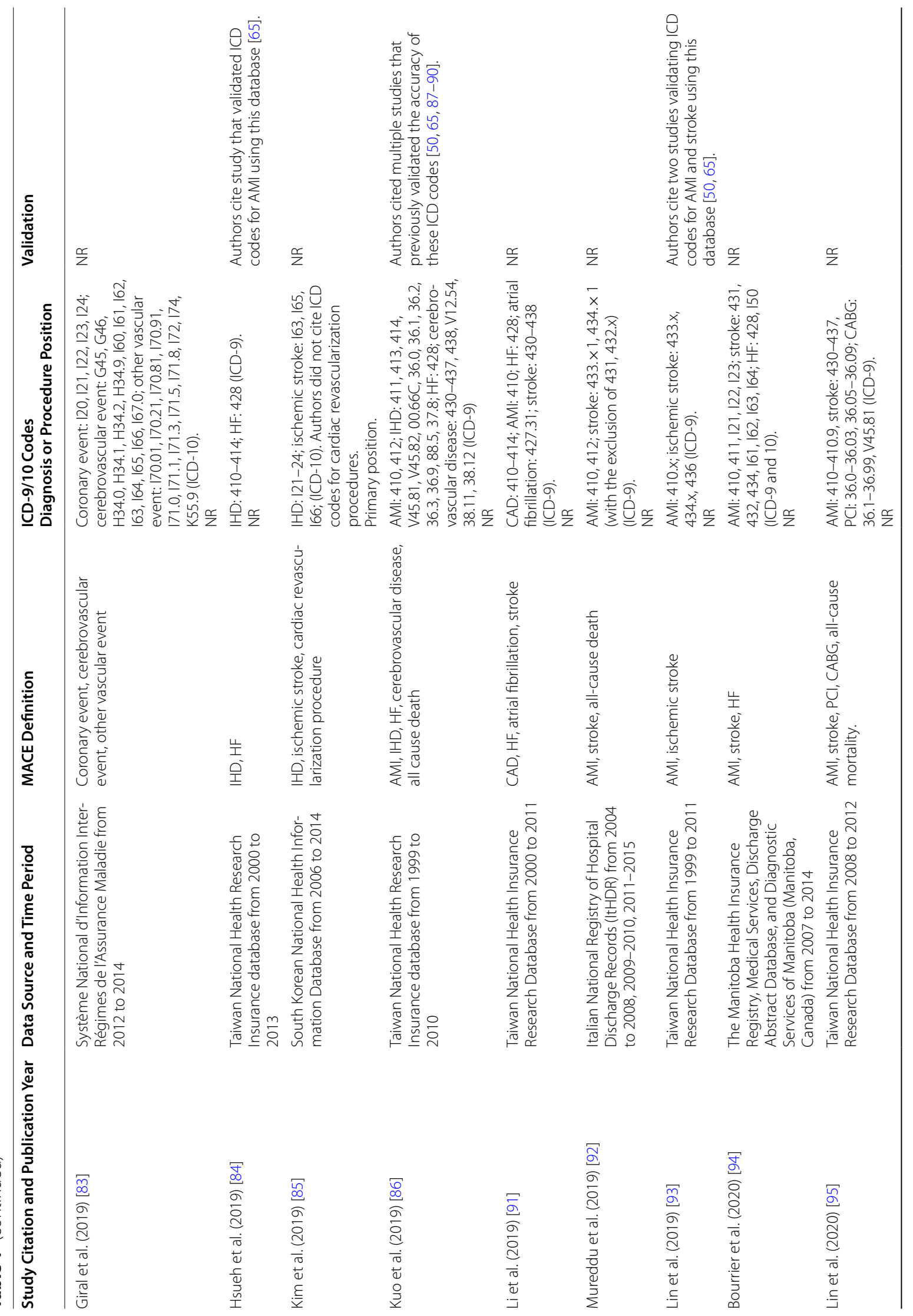




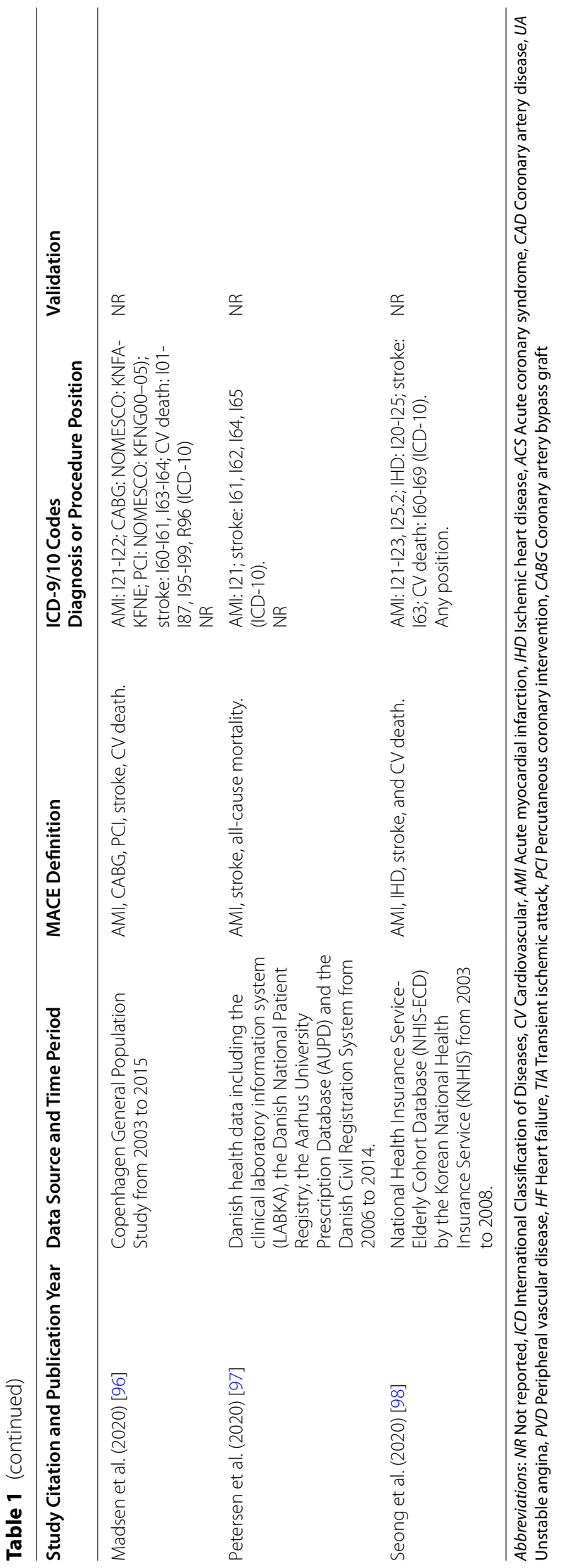


Table 2 Most common components of MACE ( $N=58$ studies)

\begin{tabular}{|c|c|c|c|}
\hline MACE Components & ICD-9 & ICD-10 & Both ICD-9/10 \\
\hline \multicolumn{4}{|l|}{ Individual components } \\
\hline $\mathrm{AMI}(n=45)$ & 29 & 14 & 2 \\
\hline Acute coronary syndrome / Ischemic Heart disease $(n=18)$ & 12 & 5 & 1 \\
\hline Stroke $(n=50)$ & 30 & 17 & 3 \\
\hline Heart failure $(n=15)$ & 12 & 2 & 1 \\
\hline All-cause death $(n=15)$ & 10 & 5 & 0 \\
\hline CV death $(n=9)$ & 1 & 6 & 2 \\
\hline Revascularization $(n=11)$ & 7 & 4 & 0 \\
\hline Other $(n=19)$ & 15 & 3 & 1 \\
\hline \multicolumn{4}{|l|}{ Combinations } \\
\hline Three-point MACE: AMI + Stroke+CV death $(n=5)$ & 1 & 3 & 1 \\
\hline Four-point MACE: AMI + Stroke+UA + CV death $(n=0)$ & 0 & 0 & 0 \\
\hline Five-point MACE: AMI + Stroke+UA + HF + CV death $(n=0)$ & 0 & 0 & 0 \\
\hline $\mathrm{AMI}+$ stroke+all-cause death $(n=8)$ & 5 & 3 & 0 \\
\hline $\mathrm{AMI}+$ stroke+HF + CV death $(n=0)$ & 0 & 0 & 0 \\
\hline $\mathrm{AMI}+$ stroke $+\mathrm{HF}+$ all-cause death $(n=2)$ & 1 & 1 & 0 \\
\hline
\end{tabular}

Abbreviations: MACE Major adverse cardiovascular events, ICD International Classification of Diseases, AMI Acute myocardial infarction, CV Cardiovascular, UA Unstable angina, $H F$ Heart failure

definitions were "AMI and stroke", and "AMI, stroke, and all-cause death," but they only made up 15.5 and $13.8 \%$ of studies, respectively. Compared to MACE definitions used in RCTs, only $8.6 \%$ of included observational studies had definitions aligned with threepoint MACE and none match four-point or five-point MACE. A large majority of studies included AMI and stroke but there was a lack of consensus with other components included in composite MACE endpoints. For instance, over half of included publications defined MACE using a definition that only was concordant with up to one other study. This diversity makes it challenging to compare findings across studies or to aggregate multiple study results for meta-analyses or systematic reviews when considering different treatment or research questions. Addressing the heterogeneity of MACE definitions used in practice requires attention due to the advantages of the MACE endpoint. Utilizing MACE as a composite outcome can potentially reduce the number of patients that need to be enrolled or identified in a retrospective cohort study, and reduce the follow-up time necessary to observe differences between different treatment groups [99]. These benefits not only potentially reduce research costs but can more expediently answer clinical questions, leading to improved patient care. Therefore, given that MACE endpoints will likely see increasing use as time goes on, there should be a continued effort to standardize and transparently report MACE definitions used in observational studies.
In 2007, findings similar to our study were observed when comparing prospective trials conducted for percutaneous coronary interventions [100]. However, although the FDA in 2008 and the EMA in 2012 attempted to standardize the MACE endpoint definition for RCTs, we found a continued discordance between how observational studies define MACE. Furthermore, we found that the majority of studies did not include mention of the diagnosis position used to define outcomes. Lack of this information prevents the ability to distinguish between incident and prevalent outcomes. While the primary position has been historically used in observational studies to define incident outcomes, the use of only primary diagnosis positions, compared to using secondary positions, may underestimate the rates of MACE for prevalent conditions [101]. The lack of reporting of diagnosis position prevents the ability to make this distinction and prohibits the full interpretation of findings.

Table 3 Most commonly used MACE components

\begin{tabular}{ll}
\hline MACE Components & Frequency, $\mathbf{n}$ (\%) \\
\hline AMI, stroke & $9(15.5)$ \\
AMI, stroke, all-cause death & $8(13.8)$ \\
AMI, stroke, CV death & $5(8.6)$ \\
ACS/IHD, stroke & $3(5.2)$ \\
AMI, stroke, HF & $3(5.2)$ \\
Other definitions ( $\leq 2$ studies using each) & $30(51.7)$ \\
\hline
\end{tabular}

Abbreviations: MACE Major adverse cardiovascular events, AMI Acute myocardial infarction, CV Cardiovascular, ACS Acute coronary syndrome, IHD Ischemic heart disease, $H F$ Heart failure 
Table 4 ICD codes by clinical outcome

\begin{tabular}{|c|c|c|c|}
\hline Outcome & ICD-9 code & ICD-10 Code & Both ICD-9/10 Codes \\
\hline $\operatorname{AMI}(n=45)$ & $\begin{array}{l}\text { 1. } 410 . x x(n=21) \\
\text { 2. } 410 . x x, 412 . x x(n=3) \\
\text { 3. Other }(n=5)\end{array}$ & $\begin{array}{l}\text { 1. } 121 . x x, 122 . x x(n=5) \\
\text { 2. } 121 . x x(n=4) \\
\text { 3. Other }(n=5)\end{array}$ & $\begin{array}{l}\text { 1. } 410 . x x, 121 . x x, 122 . x x(n=1) \\
\text { 2. 410-411.xx, } 121-123 . x x(n=1)\end{array}$ \\
\hline $\mathrm{ACS} / \mathrm{HD}(n=18)$ & $\begin{array}{l}\text { 1. } 410 . x x-414 . x x(n=3) \\
\text { 2. } 410 . x x, 411 . x x(n=2) \\
\text { 3. Other }(n=7)\end{array}$ & $\begin{array}{l}\text { 1. } 121 . x x-124 . x x(n=2) \\
\text { 2. Other }(n=3)\end{array}$ & 1. 410.xx, 411.xx, 120.0, 121.xx, 122.xx $(n=1)$ \\
\hline Stroke $(n=50)$ & $\begin{array}{l}\text { 1. } 433 . x x, 434 . x x, 436 . x x(n=4) \\
\text { 2. } 430 . x x-437 . x x(n=4) \\
\text { 3. Other }(n=22)\end{array}$ & $\begin{array}{l}\text { 1. } 163 . x x, 165 . x x, 166 . x x(n=3) \\
\text { 2. } 163 . x x(n=3) \\
\text { 3. Other }(n=11)\end{array}$ & $\begin{array}{l}\text { 2. 430.xx, 431.xx, 434.xx, 436.xx, 160.xx, 161.xx, 163.3-163.9, } 166 . \\
\text { xx }(n=1) \\
\text { 3. 433.xx-436, excluding 434.9x, 163, 164, G45 }(n=1) \\
\text { 4. 431.xx-432.xx, 434.xx, 161.xx-164.xx }(n=1)\end{array}$ \\
\hline Heart Failure $(n=15)$ & $\begin{array}{l}\text { 1. } 428 . x x(n=6) \\
\text { 2. Other }(n=6)\end{array}$ & $\begin{array}{l}\text { 1. I11.0, I50.xx, } 197.1(n=1) \\
\text { 2. } 150 . x x, K 76.1,197.1,111.0(n=1)\end{array}$ & 1. $428 . x x, 150 . x x(n=1)$ \\
\hline CV Death $(n=9)$ & 1. None listed $(n=1)$ & $\begin{array}{l}\text { 1. } 100 . x x-199 . x x(n=2) \\
\text { 2. } 146.1,146.9(n=1) \\
\text { 3. Other or none listed }(n=3)\end{array}$ & $\begin{array}{l}\text { 1. 411.xx-414.xx, 415.xx-417.xx, 420.xx-427.xx, 428.xx, 429. } \\
\text { xx, 432.xx, 433.xx, 435.xx, 437.xx, 785.51, I20.xx, I23.xx-125.xx, } \\
\text { 146.9, I30.xx-152.xx, 162.xx, 163.0-163.2, 164.xx, 165.xx, 167.xx, 168. } \\
\text { xx, R57.0 }(n=1) \\
\text { 2. 410.xx-414.xx, 428.xx, 430.xx-438.xx, 120.xx-125.xx, I50.xx, } \\
\text { 160.xx-169.xx }(n=1)\end{array}$ \\
\hline
\end{tabular}

Abbreviations: ICD International Classification of Diseases, AMI Acute myocardial infarction, ACS Acute Coronary Syndrome, IHD Ischemic Heart Disease,

CV Cardiovascular

Heterogeneity not only existed in defining MACE components but also in which ICD-9-CM and ICD-10-CM codes were used to define each individual MACE component. In our study, none of the top five components of MACE had a consensus ICD-9-CM or ICD-10-CM code that was used among all of the studies. These discrepancies contribute additional variability to our findings, as outcome classification, assuming a standard definition, inherently relies on the providers and medical billers accurately coding diseases. For example, in a study conducted in 11 Canadian emergency departments, the authors reported about $82-86 \%$ of agreement with regards to coding hospital conditions [102]. Additionally, a study looking at stroke data collected through the United States Paul Coverdell National Acute Stroke Program found that discordance existed with how strokes were coded, especially at smaller hospitals ( $<200$ beds) and when differentiating between ischemic strokes, transient ischemic attacks, and subarachnoid and intracerebral hemorrhages [103].

There have been many attempts to validate diagnosis or procedure codes for various MACE component outcomes $[32,104]$. Research has predominantly focused on the older ICD-9-CM codes, particularly studies using U.S. administrative data, but studies utilizing ICD-10-CM have more recently been published. Using data from the Centers for Medicare and Medicaid Services, a 2018 study found that the most accurate ICD-9-CM code for coding AMI was 410.xx, with a positive predictive value of $67 \%$ [104]. Additionally, the U.S. FDA commissioned the Mini-Sentinel pilot program to systematically assess the validation of common healthcare outcomes in administrative databases [32]. These studies were important steps to identify standard outcome definitions for use across disparate data sources and populations. As part of the program, systematic literature reviews from the U.S. and Canada found that the most accurate ICD9-CM code for heart failure was 428.xx, with a positive predictive value of $84-100 \%$, while the most accurate ICD-9-CM codes for stroke were 430.x, 431.x and 434.x, all with separate positive predictive values $>80 \%$, and $436 . x$, which had a positive predictive value $>70 \%[32,33]$. The Mini-Sentinel program also assessed the ICD-9-CM codes, $410 . \times 0$ or $410 . \times 1$, for AMI and found a positive predictive value of 76.394.3\% [105]. Fewer validations exist for ICD-10-CM codes. One study conducted in Japan attempted to validate the ICD-10-CM code I21.x for AMI and found a positive predictive value of $82.5 \%$ [106]. Another study in Canada found the positive predictive value for correctly coding stroke was $92 \%$ for the ICD-10-CM codes, I60.x-I61.x, I63.x-I64.x, H34.1 and G45.x [69]. Based on our findings, the most common ICD-9-CM and 10-CM codes used for AMI were also the most validated, 410.xx and I21.xx, respectively. The most validated and most common ICD-9-CM code used for heart failure was 428.xx. On the other hand, stroke had some discrepancies when comparing the most validated versus the most commonly used ICD codes. This discordance likely existed because of the differences with which stroke can be defined, 
particularly whether to include acute ischemic strokes with transient ischemic attacks, intracerebral hemorrhages or subarachnoid hemorrhages [69].

There are several limitations to our study. We excluded papers published before 2010, prior to the first published reviews of the Mini-Sentinel program, in order to present a contemporary review of the subject [32]. However, this cutoff likely skewed our results towards more modern MACE definitions and excluded different definitions that were potentially used for older publications. Older studies likely exhibited greater variety in the outcome definitions used. Additionally, because the words "major adverse cardiovascular events" were rarely explicitly written, we had to include any composite definitions that were thought to represent MACE but were listed using another composite name, such "acute cardiovascular events," based on the discretion of the reviewer. This method potentially excluded studies that used variations of MACE endpoints, but did not conform to similar naming conventions.

\section{Conclusion}

Significant heterogeneity exists in how MACE is defined and which ICD-9-CM and 10-CM codes are used to represent each respective MACE outcome in observational studies using administrative databases. The utility of future studies will improve with the use of validated definitions of AMI, stroke, cardiovascular mortality, unstable angina, and HF for the evaluation of MACE outcomes. Further, investigators should ensure that both the ICD diagnosis codes and the code positions are reported in a transparent way. Given the significant heterogeneity that already exists across administrative databases, we recommend the use of more standardized MACE definitions and corresponding ICD-9-CM and ICD-10-CM codes. This practice will allow researchers to more accurately compare findings across studies improve the reproducibility of observational studies, and decrease the potential for misleading conclusions.

\footnotetext{
Abbreviations

ACS: Acute coronary syndrome; ACS/IHD: Acute coronary syndrome or ischemic heart disease; AMl: Acute myocardial infarction; CABG: Coronary artery bypass graft; CAD: Coronary artery disease; CV: Cardiovascular; HF: Heart failure; ICD: International Classification of Diseases; ICD-9-CM: International Classification of Diseases, 9th Revision, Clinical Modification; ICD-10-CM: International Classification of Diseases, 10th Revision, Clinical Modification; IHD: Ischemic heart disease; MACE: Major adverse cardiovascular events; PCl: Percutaneous coronary intervention; PRISMA: Preferred Reporting Items for Systematic Reviews and Meta-Analysis; PRISMA-P: Preferred Reporting Items for Systematic Reviews and Meta-Analysis Protocols; PROSPERO: International Prospective Register of Systematic Reviews; PVD: Peripheral vascular disease; RCT: Randomized controlled trial; TIA: Transient ischemic attack; UA: Unstable angina.
}

\section{Supplementary Information}

The online version contains supplementary material available at https://doi. org/10.1186/s12874-021-01440-5.

Additional file 1: Supplementary Text 1. Systematic Review Protocol.

Additional file 2: Supplementary Table 1. PRISMA 2020 Checklist.

Additional file 3: Supplementary Text 2. Search terms.

\section{Acknowledgements}

Not applicable.

\section{Authors' contributions}

E.B., L.H., E.S., K.W.M, and S.G. took part in conceiving the initial plans of the study. E.B., L.H., and E.S. developed the search criteria, reviewing abstracts and full texts, extracted study data, and prepared the initial draft of the manuscript. E.B., L.H., E.S., K.W.M, and S.G took part in analyzing and interpreting the results, providing critical revisions for the manuscript, and approving the final manuscript.

Funding

Unfunded.

Availability of data and materials

Data sharing is not applicable to this article as no datasets were generated or analyzed during the current study.

\section{Declarations}

Ethics approval and consent to participate

Not applicable.

\section{Consent for publication \\ Not applicable.}

\section{Competing interests}

E.B., L.H., and E.S. declare no competing interests. K.W.M reports investigatorinitiated grants from Seqirus, Pfizer and Sanofi Pasteur. S.G. reports grants from Seqirus, Sanofi; and consulting or speaker fees from Sanofi, Seqirus, Merck, and the Gerontological Society of America related to vaccines or nursing home care quality. The content and views expressed in this article are those of the authors and do not necessarily reflect the position or policy of the Department of Veterans Affairs or the United States government.

\section{Author details}

${ }^{1}$ Department of Health Services, Policy, and Practice, Brown University School of Public Health, 121 South Main Street, Box G-S121-3, Providence, RI 02912, USA. ${ }^{2}$ Center for Gerontology and Healthcare Research, Brown University School of Public Health, RI, Providence, USA. ${ }^{3}$ Department of Medicine, Warren Alpert Medical School of Brown University, Providence, RI, USA. ${ }^{4}$ Center of Innovation in Long-Term Services and Supports, Providence Veterans Affairs Medical Center, Providence, RI, USA. ${ }^{5}$ Division of Infectious Diseases and HIV Medicine, University Hospitals Cleveland Medical Center, Cleveland, OH, USA.

${ }^{6}$ School of Medicine, Case Western Reserve University, Cleveland, OH, USA.

Received: 15 June 2021 Accepted: 12 October 2021

Published online: 06 November 2021

\section{References}

1. Gal D, Thijs B, Glanzel W, Sipido KR. Hot topics and trends in cardiovascular research. Eur Heart J. 2019;40(28):2363-74.

2. Benjamin EJ, Muntner P, Alonso A, Bittencourt MS, Callaway CW, Carson AP, et al. Heart disease and stroke statistics-2019 update: a report from the American Heart Association. Circulation. 2019;139(10):e56-e528.

3. Sharma A, Pagidipati NJ, Califf RM, McGuire DK, Green JB, Demets D, et al. Impact of regulatory guidance on evaluating cardiovascular risk 
of new glucose-lowering therapies to treat type 2 diabetes mellitus: lessons learned and future directions. Circulation. 2020;141(10):843-62.

4. Marx N, McGuire DK, Perkovic V, Woerle HJ, Broedl UC, von Eynatten M, et al. Composite primary end points in cardiovascular outcomes trials involving type 2 diabetes patients: should unstable angina be included in the primary end point? Diabetes Care. 2017;40(9):1144-51.

5. Gavrielov-Yusim N, Friger M. Use of administrative medical databases in population-based research. J Epidemiol Community Health. 2014:68(3):283-7.

6. Franklin JM, Schneeweiss $\mathrm{S}$. When and how can real world data analyses substitute for randomized controlled trials? Clin Pharmacol Ther. 2017;102(6):924-33.

7. Wang SV, Pinheiro S, Hua W, Arlett P, Uyama Y, Berlin JA, et al. STaRTRWE: structured template for planning and reporting on the implementation of real world evidence studies. BMJ (Clinical research ed). 2021;372:m4856

8. Franklin JM, Patorno E, Desai RJ, Glynn RJ, Martin D, Quinto K, et al. Emulating randomized clinical trials with nonrandomized real-world evidence studies: first results from the RCT DUPLICATE Initiative. Circulation. 2021;143(10):1002-13.

9. International Statistical Classification of Diseases and Related Health Problems (ICD). World Health Organization; 2021. https://www.who. int/standards/classifications/classification-of-diseases. Accessed 28 Feb 2021

10. McCormick N, Bhole V, Lacaille D, Avina-Zubieta JA. Validity of diagnostic codes for acute stroke in administrative databases: a systematic review. PLoS One. 2015;10(8):e0135834.

11. McCormick N, Lacaille D, Bhole V, Avina-Zubieta JA. Validity of myocardial infarction diagnoses in administrative databases: a systematic review. PLoS One. 2014;9(3):e92286.

12. McCormick N, Lacaille $D$, Bhole V, Avina-Zubieta JA. Validity of heart failure diagnoses in administrative databases: a systematic review and meta-analysis. PLoS One. 2014;9(8):e104519.

13. Moher D, Shamseer L, Clarke M, Ghersi D, Liberati A, Petticrew M, et al. Preferred reporting items for systematic review and meta-analysis protocols (PRISMA-P) 2015 statement. Syst Rev. 2015;4:1.

14. Page MJ, McKenzie JE, Bossuyt PM, Boutron I, Hoffmann TC, Mulrow CD, et al. The PRISMA 2020 statement: an updated guideline for reporting systematic reviews. BMJ (Clinical research ed). 2021;372:n71.

15. Wallace B, Small K, Brodley CE, Lau J, Trikalinos TA. Deploying an interactive machine learning system in an evidence-based practice center: abstrackr. In: Proceedings of the ACM International Health Informatics Symposium (IHI); 2012. p. 819-24.

16. Chapman RH, Yeaw J, Roberts CS. Association between adherence to calcium-channel blocker and statin medications and likelihood of cardiovascular events among US managed care enrollees. BMC Cardiovasc Disord. 2010;10:29.

17. Gudbjornsson B, Thorsteinsson SB, Sigvaldason H, Einarsdottir R, Johannsson M, Zoega H, et al. Rofecoxib, but not celecoxib, increases the risk of thromboembolic cardiovascular events in young adults-a nationwide registry-based study. Eur J Clin Pharmacol. 2010;66(6):619-25.

18. Charlot M, Grove EL, Hansen PR, Olesen JB, Ahlehoff O, Selmer C, et al. Proton pump inhibitor use and risk of adverse cardiovascular events in aspirin treated patients with first time myocardial infarction: nationwide propensity score matched study. BMJ (Clinical research ed). 2011;342:d2690.

19. Madsen M, Davidsen M, Rasmussen S, Abildstrom SZ, Osler M. The validity of the diagnosis of acute myocardial infarction in routine statistics: a comparison of mortality and hospital discharge data with the Danish MONICA registry. J Clin Epidemiol. 2003;56(2):124-30.

20. Krarup LH, Boysen G, Janjua H, Prescott E, Truelsen T. Validity of stroke diagnoses in a National Register of Patients. Neuroepidemiology. 2007:28(3):150-4.

21. Kociol RD, Hammill BG, Hernandez AF, Klaskala W, Mills RM, Curtis LH, et al. Pharmacologic prophylaxis for venous thromboembolism and 30-day outcomes among older patients hospitalized with heart failure: an analysis from the ADHERE national registry linked to Medicare claims. Clin Cardiol. 2011;34(11):682-8.
22. Chen ZY, Chiang CH, Huang CC, Chung CM, Chan WL, Huang PH, et al. The association of tooth scaling and decreased cardiovascular disease: a nationwide population-based study. Am J Med. 2012;125(6):568-75.

23. Chen HJ, Bai CH, Yeh WT, Chiu HC, Pan WH. Influence of metabolic syndrome and general obesity on the risk of ischemic stroke. Stroke. 2006;37(4):1060-4.

24. Degli Esposti L, Saragoni S, Batacchi P, Benemei S, Geppetti P, Sturani $A$, et al. Adherence to statin treatment and health outcomes in an Italian cohort of newly treated patients: results from an administrative database analysis. Clin Ther. 2012;34(1):190-9.

25. Parker ED, Margolis KL, Trower NK, Magid DJ, Tavel HM, Shetterly $S M$, et al. Comparative effectiveness of $2 \beta$-blockers in hypertensive patients. Arch Intern Med. 2012;172(18):1406-12.

26. Karthikesalingam A, Bahia SS, Patterson BO, Peach G, Vidal-Diez A, Ray $\mathrm{KK}$, et al. The shortfall in long-term survival of patients with repaired thoracic or abdominal aortic aneurysms: retrospective case-control analysis of hospital episode statistics. Eur J Vasc Endovasc Surg. 2013:46(5):533-41.

27. Mortensen EM, Halm EA, Pugh MJ, Copeland LA, Metersky M, Fine MJ, et al. Association of azithromycin with mortality and cardiovascular events among older patients hospitalized with pneumonia. JAMA. 2014;311(21):2199-208.

28. Neumann A, Maura G, Weill A, Ricordeau P, Alla F, Allemand H. Comparative effectiveness of rosuvastatin versus simvastatin in primary prevention among new users: a cohort study in the French national health insurance database. Pharmacoepidemiol Drug Saf. 2014;23(3):240-50.

29. Vanasse A, Courteau J, Asghari S, Leroux D, Cloutier L. Health inequalities associated with neighbourhood deprivation in the Quebec population with hypertension in primary prevention of cardiovascular disease. Chronic Dis Inj Can. 2014;34(4):181-94

30. Chou SH, Tung YC, Lin YS, Wu LS, Lin CP, Liou EJ, et al. Major adverse cardiovascular events in treated periodontitis: a population-based follow-up study from Taiwan. PLoS One. 2015;10(6):e0130807.

31. Desai JR, Vazquez-Benitez G, Xu Z, Schroeder EB, Karter AJ, Steiner $J F$, et al. Who must we target now to minimize future cardiovascular events and total mortality?: lessons from the surveillance, prevention and management of diabetes mellitus (SUPREME-DM) cohort study. Circ Cardiovas Qual Outcomes. 2015;8(5):508-16.

32. Saczynski JS, Andrade SE, Harrold LR, Tjia J, Cutrona SL, Dodd KS, et al. A systematic review of validated methods for identifying heart failure using administrative data. Pharmacoepidemiol Drug Saf. 2012;21 (Suppl 1):129-40.

33. Andrade SE, Harrold LR, Tjia J, Cutrona SL, Saczynski JS, Dodd KS, et al. A systematic review of validated methods for identifying cerebrovascular accident or transient ischemic attack using administrative data. Pharmacoepidemiol Drug Saf. 2012;21(Suppl 1):100-28.

34. Yeh RW, Sidney S, Chandra M, Sorel M, Selby JV, Go AS. Population trends in the incidence and outcomes of acute myocardial infarction. N Engl J Med. 2010;362(23):2155-65.

35. Doll JA, Hellkamp A, Thomas L, Ho PM, Kontos MC, Whooley MA, et al. Effectiveness of cardiac rehabilitation among older patients after acute myocardial infarction. Am Heart J. 2015;170(5):855-64.

36. Jernberg T, Hasvold P, Henriksson M, Hjelm H, Thuresson M, Janzon M. Cardiovascular risk in post-myocardial infarction patients: nationwide real world data demonstrate the importance of a long-term perspective. Eur Heart J. 2015;36(19):1163-70.

37. Linnersjö A, Hammar N, Gustavsson A, Reuterwall C. Recent time trends in acute myocardial infarction in Stockholm, Sweden. Int J Cardiol. 2000;76(1):17-21.

38. Korsnes JS, Davis KL, Ariely R, Bell CF, Mitra D. Health care resource utilization and costs associated with nonfatal major adverse cardiovascular events. J Manag Care Spec Pharm. 2015;21(6):443-50.

39. Roussel R, Chaignot C, Weill A, Travert F, Hansel B, Marre M, et al. Use of fibrates monotherapy in people with diabetes and high cardiovascular risk in primary care: a French nationwide cohort study based on national administrative databases. PLoS One. 2015;10(9):e0137733.

40. Sirois C, Moisan J, Poirier P, Gregoire JP. Comparative effectiveness of cardioprotective drugs in elderly individuals with type 2 diabetes. Int J Clin Pract. 2015;69(3):305-12. 
41. Levy AR, Tamblyn RM, Fitchett D, McLeod PJ, Hanley JA. Coding accuracy of hospital discharge data for elderly survivors of myocardial infarction. Can J Cardiol. 1999;15(11):1277-82.

42. Stochkendahl MJ, Mickley H, Vach W, Aziz A, Christensen HW, Hartvigsen J, et al. Clinical characteristics, myocardial perfusion deficits, and clinical outcomes of patients with non-specific chest pain hospitalized for suspected acute coronary syndrome: a 4-year prospective cohort study. Int J Cardiol. 2015;182:126-31.

43. Blin P, Philippe F, Bouee S, Laurendeau C, Torreton E, Gourmelin J, et al. Outcomes following acute hospitalised myocardial infarction in France: an insurance claims database analysis. Int J Cardiol. 2016;219:387-93.

44. Cheng CA, Chien WC, Hsu CY, Lin HC, Chiu HW. Risk analysis of carotid stent from a population-based database in Taiwan. Medicine. 2016:95(35):e4747.

45. Chuang CS, Ho SC, Lin CL, Lin MC, Kao CH. Risk of cerebrovascular events in pneumoconiosis patients: a population-based study, 19962011. Medicine. 2016;95(9):e2944.

46. Fortuna LA, Pawloski PA, Parker ED, Trower NK, Kottke TE. Proton pump inhibitor use by aspirin-treated coronary artery disease patients is not associated with increased risk of cardiovascular events. Eur Heart J Cardiovasc Pharmacother. 2016;2(1):13-9.

47. Kottke TE, Baechler CJ, Parker ED. Accuracy of heart disease prevalence estimated from claims data compared with an electronic health record. Prev Chronic Dis. 2012;9:E141.

48. Hsu CY, Chen YT, Huang PH, Leu HB, Su YW, Chiang CH, et al. The association between urinary calculi and increased risk of future cardiovascular events: a nationwide population-based study. J Cardiol. 2016;67(5):463-70.

49. Lindblad U, Råstam L, Ranstam J, Peterson M. Validity of register data on acute myocardial infarction and acute stroke: the Skaraborg hypertension project. Scand J Soc Med. 1993;21(1):3-9.

50. Cheng C-L, Kao Y-HY, Lin S-J, Lee C-H, Lai ML. Validation of the national health insurance research database with ischemic stroke cases in Taiwan. Pharmacoepidemiol Drug Saf. 2011;20(3):236-42.

51. Shih C-J, Chen H-T, Chao P-W, Kuo S-C, Li S-Y, Yang C-Y, et al. Angiotensin-converting enzyme inhibitors, angiotensin II receptor blockers and the risk of major adverse cardiac events in patients with diabetes and prior stroke: a nationwide study. J Hypertens. 2016;34(3):567-75.

52. Hsieh CY, Chen CH, Li CY, Lai ML. Validating the diagnosis of acute ischemic stroke in a National Health Insurance claims database. J Formos Med Assoc. 2015;114(3):254-9.

53. Solomon DH, Liu CC, Kuo IH, Zak A, Kim SC. Effects of colchicine on risk of cardiovascular events and mortality among patients with gout: a cohort study using electronic medical records linked with Medicare claims. Ann Rheum Dis. 2016;75(9):1674-9.

54. Kiyota Y, Schneeweiss S, Glynn RJ, Cannuscio CC, Avorn J, Solomon $\mathrm{DH}$. Accuracy of Medicare claims-based diagnosis of acute myocardial infarction: estimating positive predictive value on the basis of review of hospital records. Am Heart J. 2004;148(1):99-104.

55. Birman-Deych E, Waterman AD, Yan Y, Nilasena DS, Radford MJ, Gage BF. Accuracy of ICD-9-CM codes for identifying cardiovascular and stroke risk factors. Med Care. 2005;43(5):480-5.

56. Babazade R, Yilmaz HO, Leung SM, Zimmerman NM, Turan A. Systemic lupus erythematosus is associated with increased adverse postoperative renal outcomes and mortality: a historical cohort study using administrative health data. Anesth Analg. 2017;124(4):1118-26.

57. de Miguel-Yanes JM, Jimenez-Garcia R, Hernandez-Barrera V, MendezBailon M, de Miguel-Diez J, Lopez-de-Andres A. Impact of type 2 diabetes mellitus on in-hospital-mortality after major cardiovascular events in Spain (2002-2014). Cardiovasc Diabetol. 2017;16(1):126.

58. Lindahl B, Baron T, Erlinge D, Hadziosmanovic N, Nordenskjöld A, Gard $A$, et al. Medical therapy for secondary prevention and long-term outcome in patients with myocardial infarction with nonobstructive coronary artery disease. Circulation. 2017;135(16):1481-9.

59. Policardo L, Seghieri G, Francesconi P, Anichini R, Franconi F, Del Prato S. Gender difference in diabetes related excess risk of cardiovascular events: when does the 'risk window' open? J Diabetes Complicat. 2017:31(1):74-9.

60. Tung YC, Huang YC, Wu LS, Chang CJ, Chu PH. Medication compliance and clinical outcomes of fixed-dose combinations vs free combinations of an angiotensin II receptor blocker and a calcium channel blocker in hypertension treatment. J Clin Hypertens (Greenwich). 2017;19(10):983-9.

61. Arinze N, Farber A, Sachs T, Patts G, Kalish J, Kuhnen A, et al. The effect of statin use and intensity on stroke and myocardial infarction after carotid endarterectomy. J Vasc Surg. 2018;68(5):1398-405.

62. Baviera M, Bertele V, Avanzini F, Vannini T, Tettamanti M, Fortino I, et al. Peripheral arterial disease: Changes in clinical outcomes and therapeutic strategies in two cohorts, from 2002 to 2008 and from 2008 to 2014. A population-based study. Eur J Prev Cardiol. 2018;25(16):1735-43.

63. Chan CW, Yu CL, Lin JC, Hsieh YC, Lin CC, Hung CY, et al. Glitazones and alpha-glucosidase inhibitors as the second-line oral anti-diabetic agents added to metformin reduce cardiovascular risk in type 2 diabetes patients: a nationwide cohort observational study. Cardiovasc Diabetol. 2018;17(1):20.

64. Wu VC, Chen SW, Ting PC, Chang CH, Wu M, Lin MS, et al. Selection of $\beta$-blocker in patients with cirrhosis and acute myocardial infarction: a 13-year nationwide population-based study in Asia. J Am Heart Assoc. 2018;7(19):e008982.

65. Cheng CL, Lee CH, Chen PS, Li YH, Lin SJ, Yang YH. Validation of acute myocardial infarction cases in the national health insurance research database in Taiwan. J Epidemiol. 2014;24(6):500-7.

66. Degli Esposti L, Perrone V, Veronesi C, Buda S, Rossini R. All-cause mortality, cardiovascular events, and health care costs after 12 months of dual platelet aggregation inhibition after acute myocardial infarction in real-world patients: findings from the platelet-aggregation inhibition: persistence with treatment and cardiovascular events in real world (PIPER) study. Vasc Health Risk Manag. 2018;14:383-92.

67. Hussain MA, Saposnik G, Raju S, Salata K, Mamdani M, Tu $\mathrm{JV}$, et al. Association between statin use and cardiovascular events after carotid artery revascularization. J Am Heart Assoc. 2018;7(16):e009745.

68. Juurlink D, Preyra C, Croxford R, Chong A, Austin P, Tu J, et al. Canadian institute for health information discharge abstract database: a validation study. Toronto: ICES investigative report Institute for Clinical Evaluative Sciences; 2006.

69. Kokotailo RA, Hill MD. Coding of stroke and stroke risk factors using international classification of diseases, revisions 9 and 10. Stroke. 2005;36(8):1776-81.

70. Jin P, Matos Diaz I, Stein L, Thaler A, Tuhrim S, Dhamoon MS. Intermediate risk of cardiac events and recurrent stroke after stroke admission in young adults. Int J Stroke. 2018;13(6):576-84.

71. Goldstein LB. Accuracy of ICD-9-CM coding for the identification of patients with acute ischemic stroke: effect of modifier codes. Stroke. 1998;29(8):1602-4.

72. Roumie CL, Mitchel E, Gideon PS, Varas-Lorenzo C, Castellsague J, Griffin MR. Validation of ICD-9 codes with a high positive predictive value for incident strokes resulting in hospitalization using Medicaid health data. Pharmacoepidemiol Drug Saf. 2008;17(1):20-6.

73. Kim D, Yang PS, Kim TH, Jang E, Shin H, Kim HY, et al. Ideal blood pressure in patients with atrial fibrillation. J Am Coll Cardiol. 2018;72(11):1233-45.

74. Kiss Z, Rokszin G, Abonyi-Toth Z, Jermendy G, Kempler P, Aradi D, et al. Dissimilar impact of type 2 diabetes on cardiovascular outcomes according to age categories: a nationwide population study from Hungary. Cardiovasc Diabetol. 2018;17(1):107.

75. Ohm J, Skoglund PH, Discacciati A, Sundstrom J, Hambraeus K, Jernberg T, et al. Socioeconomic status predicts second cardiovascular event in 29,226 survivors of a first myocardial infarction. Eur J Prev Cardiol. 2018;25(9):985-93.

76. Winell K, Arffman M, Pietila A, Salomaa V. Regional differences in the incidence of diabetic cardiovascular events reflect the quality of care. Scand Cardiovasc J. 2018;52(5):232-7.

77. Pajunen P, Koukkunen $H$, Ketonen $M$, Jerkkola T, Immonen-Räihä P, Kärjä-Koskenkari P, et al. The validity of the Finnish hospital discharge register and causes of death register data on coronary heart disease. Eur J Cardiovasc Prev Rehabil. 2005;12(2):132-7.

78. Tolonen H, Salomaa V, Torppa J, Sivenius J, Immonen-Räihä P, Lehtonen A. The validation of the Finnish hospital discharge register and causes of death register data on stroke diagnoses. Eur J Cardiovasc Prev Rehabil. 2007;14(3):380-5. 
79. Yang CT, Kor CT, Hsieh YP. Long-term effects of spironolactone on kidney function and hyperkalemia-associated hospitalization in patients with chronic kidney disease. J Clin Med. 2018;7(11):459.

80. Young JB, Gauthier-Loiselle M, Bailey RA, Manceur AM, Lefebvre P, Greenberg $M$, et al. Development of predictive risk models for major adverse cardiovascular events among patients with type 2 diabetes mellitus using health insurance claims data. Cardiovasc Diabetol. 2018;17(1):118.

81. Arnaout R, Nah G, Marcus G, Tseng Z, Foster E, Harris IS, et al. Pregnancy complications and premature cardiovascular events among 1.6 million California pregnancies. Open Heart. 2019;6(1):e000927.

82. Chen S, Hsu WY, Lin YN, Wang CY, Wu CH, Chang KH. Incidence and risk of major adverse cardiovascular events in middle-aged patients with chronic kidney disease: a population-based cohort study. Int Urol Nephrol. 2019;51(7):1219-27.

83. Giral P, Neumann A, Weill A, Coste J. Cardiovascular effect of discontinuing statins for primary prevention at the age of 75 years: a nationwide population-based cohort study in France. Eur Heart J. 2019;40(43):3516-25.

84. Hsueh CM, Wey JH, Yeh JS, Wu CH, Liou TH, Chang KH. Incidence and risk of major heart diseases in middle-aged adults with moderate to severe vision impairment: a population-based cohort study. Br J Ophthalmol. 2019;103(8):1054-9.

85. Kim K, Kwak A, Choi CU, Kim JH, Kim MG, Oh JM, et al. Differences in preventing new-onset cardiovascular events with statin therapy in seniors aged 75 years and over: a cohort study in the South Korean National Health Insurance Database. Basic Clin Pharmacol Toxicol. 2019;125(2):108-16.

86. Kuo S, Yang CT, Wu JS, Ou HT. Effects on clinical outcomes of intensifying triple oral antidiabetic drug (OAD) therapy by initiating insulin versus enhancing OAD therapy in patients with type 2 diabetes: a nationwide population-based, propensity-score-matched cohort study. Diabetes Obes Metab. 2019;21(2):312-20.

87. Chang CH, Lee YC, Tsai CT, Chang SN, Chung YH, Lin MS, et al. Continuation of statin therapy and a decreased risk of atrial fibrillation/flutter in patients with and without chronic kidney disease. Atherosclerosis. 2014;232(1):224-30.

88. Lin YS, Chen TH, Chi CC, Lin MS, Tung TH, Liu CH, et al. Different implications of heart failure, ischemic stroke, and mortality between nonvalvular atrial fibrillation and atrial flutter-a view from a national cohort study. J Am Heart Assoc. 2017;6(7):e006406.

89. Wu CS, Lai MS, Gau SS, Wang SC, Tsai HJ. Concordance between patient self-reports and claims data on clinical diagnoses, medication use, and health system utilization in Taiwan. PLoS One. 2014;9(12):e112257.

90. Sung SF, Hsieh CY, Lin HJ, Chen YW, Yang YH, Li CY. Validation of algorithms to identify stroke risk factors in patients with acute ischemic stroke, transient ischemic attack, or intracerebral hemorrhage in an administrative claims database. Int J Cardiol. 2016;215:277-82.

91. Li SY, Chen HH, Lin CL, Yeh SY, Kao CH. The different cardiovascular outcomes between long-term efficacy of hydrophilic and lipophilic statin therapy in both Asian diabetic sexes. Dose Response. 2019;17(3):1559325819876766.

92. Mureddu GF, Greco C, Rosato S, D’Errigo P, De Luca L, Badoni G, et al. High thrombotic risk increases adverse clinical events up to 5 years after acute myocardial infarction. A nationwide retrospective cohort study. Monaldi Arch Chest Dis. 2019;89(3). https://doi.org/10.4081/ monaldi.2019.1166

93. Lin Y-F, Chen L, Lin S-L, Yeh Y-C, Huang T-M, Chou Y-H, et al. Potential target-organ protection of mineralocorticoid receptor antagonist in acute kidney disease. J Hypertens. 2019;37(1):125-34.
94. Bourrier M, Ferguson TW, Embil JM, Rigatto C, Komenda P, Tangri N. Peripheral artery disease: its adverse consequences with and without CKD. Am J Kidney Dis. 2020;75(5):705-12.

95. Lin CP, Tung YC, Hsiao FC, Yang CH, Kao YW, Lin YS, et al. Fixed-dose combination of amlodipine and atorvastatin improves clinical outcomes in patients with concomitant hypertension and dyslipidemia. J Clin Hypertens (Greenwich). 2020;22:1846-53.

96. Madsen CM, Kamstrup PR, Langsted A, Varbo A, Nordestgaard BG. Lipoprotein(a)-lowering by $50 \mathrm{mg} / \mathrm{dL}(105 \mathrm{nmol} / \mathrm{L})$ may be needed to reduce cardiovascular disease $20 \%$ in secondary prevention: a population-based study. Arterioscler Thromb Vasc Biol. 2020;40(1):255-66.

97. Petersen I, Nicolaisen SK, Ricciardi F, Sharma M, Thomsen RW, Baio G, et al. Impact of being eligible for type 2 diabetes treatment on all-cause mortality and cardiovascular events: regression discontinuity design study. Clin Epidemiol. 2020;12:569-77.

98. Seong JM, Shin D, Sung JW, Cho S, Yang J, Kang S, et al. Gonadotropinreleasing hormone agonists, anti-androgens and the risk of cardio-cerebrovascular disease in prostate cancer patients: an asian populationbased observational study. J Cancer. 2020;11(14):4015-22.

99. Freemantle N, Calvert M, Wood J, Eastaugh J, Griffin C. Composite outcomes in randomized trials: greater precision but with greater uncertainty? JAMA. 2003;289(19):2554-9.

100. Kip KE, Hollabaugh K, Marroquin OC, Williams DO. The problem with composite end points in cardiovascular studies: the story of major adverse cardiac events and percutaneous coronary intervention. J Am Coll Cardiol. 2008;51(7):701-7.

101. Kerr AJ, Wang TKM, Jiang Y, Grey C, Wells S, Poppe KK. The importance of considering both primary and secondary diagnostic codes when using administrative health data to study acute coronary syndrome epidemiology (ANZACS-Q| 47). Eur Heart J Qual Care Clin Outcomes. 2020;7:548-55.

102. Peng M, Eastwood C, Boxill A, Jolley RJ, Rutherford L, Carlson K, et al. Coding reliability and agreement of international classification of disease, 10(th) revision (ICD-10) codes in emergency department data. Int J Popul Data Sci. 2018:3(1):445.

103. Chang TE, Lichtman JH, Goldstein LB, George MG. Accuracy of ICD-9-CM codes by hospital characteristics and stroke severity: Paul Coverdell National Acute Stroke Program. J Am Heart Assoc. 2016;5(6):e003056.

104. Bush M, Sturmer T, Stearns SC, Simpson RJ Jr, Brookhart MA, Rosamond W, et al. Position matters: validation of medicare hospital claims for myocardial infarction against medical record review in the atherosclerosis risk in communities study. Pharmacoepidemiol Drug Saf. 2018;27(10):1085-91.

105. Cutrona SL, Toh S, Iyer A, Foy S, Daniel GW, Nair VP, et al. Validation of acute myocardial infarction in the Food and Drug Administration's Minisentinel program. Pharmacoepidemiol Drug Saf. 2013;22(1):40-54.

106. Ando T, Ooba N, Mochizuki M, Koide D, Kimura K, Lee SL, et al. Positive predictive value of ICD-10 codes for acute myocardial infarction in Japan: a validation study at a single center. BMC Health Serv Res. 2018;18(1):895.

\section{Publisher's Note}

Springer Nature remains neutral with regard to jurisdictional claims in published maps and institutional affiliations. 